\title{
Thermal creep induced by cooling a superconducting vortex lattice
}

\author{
Roland Willa $\odot,{ }^{1,2,3}$ Jose Augusto Galvis $\odot,{ }^{4}$ Jose Benito-Llorens, ${ }^{5}$ Edwin Herrera $\odot, 4,5$ \\ Isabel Guillamon $\odot,{ }^{5}$ and Hermann Suderow $\odot^{5}$ \\ ${ }^{1}$ Institute for Theoretical Physics, ETH Zurich, 8093 Zurich, Switzerland \\ ${ }^{2}$ Materials Science Division, Argonne National Laboratory, Argonne, Illinois 60439, USA \\ ${ }^{3}$ Institute of Condensed Matter Theory, Karlsruhe Institute of Technology, 76131 Karlsruhe, Germany \\ ${ }^{4}$ Departamento de Ciencias Naturales, Facultad de Ingeniería y Ciencias Básicas, Universidad Central, Bogotá, Colombia \\ ${ }^{5}$ Laboratorio de Bajas Temperaturas y Altos Campos Magnéticos, Unidad Asociada UAM/CSIC, \\ Departamento de Física de la Materia Condensada, Instituto de Ciencia de Materiales Nicolás Cabrera, \\ Condensed Matter Physics Center (IFIMAC), Universidad Autónoma de Madrid, E-28049 Madrid, Spain
}

(Received 14 June 2019; revised manuscript received 8 November 2019; accepted 22 November 2019; published 5 February 2020)

\begin{abstract}
A perturbed system relaxes towards an equilibrium given by a minimum in the potential-energy landscape. This often occurs by thermally activated jumps over metastable states. The corresponding dynamics is called creep and follows Arrhenius' law. Here we consider the situation where the equilibrium position itself depends on temperature. We show that this effect occurs in the vortex lattice of the anisotropic superconductor $2 \mathrm{H}-\mathrm{NbSe}_{2}$ when the magnetic field is tilted away from the principal axes, and that it leads to the peculiar appearance of creep when cooling the sample. Temperature determines the system's equilibrium state and at the same time brings the system back to equilibrium, playing a dual and antagonistic role. We expect that cooling-induced creep occurs in correlated systems with many degrees of freedom, allowing one to tune the equilibrium state via heat treatment.
\end{abstract}

DOI: 10.1103/PhysRevResearch.2.013125

\section{INTRODUCTION}

Superconducting vortices are lines of quantized magnetic flux $\Phi_{0}=h c / 2 e$ where the superconducting order parameter is depressed at a length scale of the order of the superconducting coherence length $\xi$ and circular supercurrents are flowing at a length scale of the order of the penetration depth $\lambda$. Vortices arrange in a lattice, which can be triangular, square, or disordered due to the interactions of vortices with the crystalline environment [1-5]. Vortices tend to be pinned on material defects or inclusions consisting of places where superconductivity is depressed on length scales of the order of $\xi$. When varying the applied magnetic field, its strength, or direction, vortices enter or exit the sample [1-3]. This produces vortex motion, which is often counteracted by pinning. The action of the pinning landscape results in long-lived out-of-equilibrium vortex distributions that relax through thermal creep over a manifold of barriers [1-3,6-10]. The phenomenon of creep has been observed in interacting systems of particles, such as colloids, polymers, solids consisting of mixtures, or in lattices of entities formed through electronic interactions (domain walls or skyrmions) [11-15]. To study pinning and creep vortex lattices are often considered as a model system [15-18]. Most efforts to understand vortex creep have focused on trying to immobilize vortices and thereby increase the critical current $j_{c}$ for applications [19]. But the interaction between vortices

Published by the American Physical Society under the terms of the Creative Commons Attribution 4.0 International license. Further distribution of this work must maintain attribution to the author(s) and the published article's title, journal citation, and DOI. and the underlying superconducting material is very rich and can produce counterintuitive phenomena. Here we find that the equilibrium state towards which the system creeps can be modified with the temperature, leading to a phenomenon that we term self-imposed creep; see Fig. 1. We study vortex creep in the superconductor $2 \mathrm{H}-\mathrm{NbSe}_{2}$ and observe that after

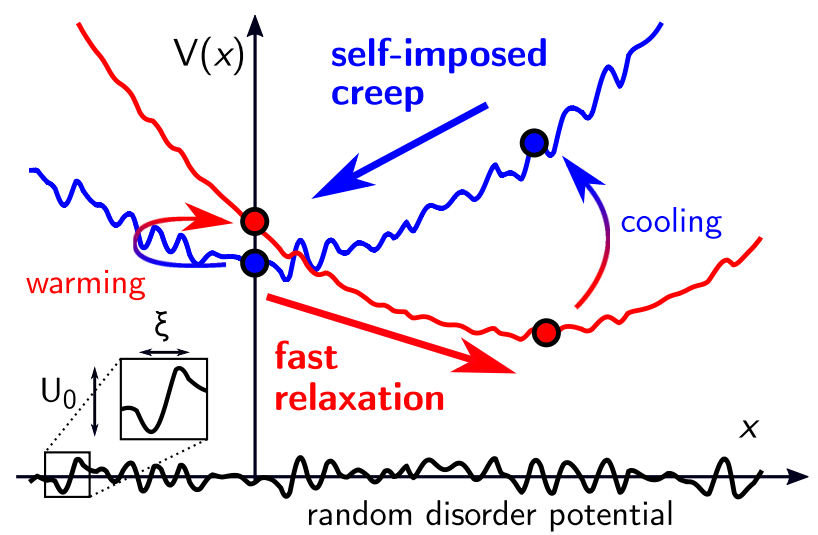

FIG. 1. Schematic response of a particle confined in a potential subject to random disorder. The disorder potential is characterized by local maxima of size $U_{0}$ and over a length scale $\xi$ (the superconducting coherence length in the case of vortices in superconductors). Upon warming, the particle rapidly moves to a newly defined minimum as the disorder is thermally smeared out [indicated by effectively smaller wells (red)]. When cooling, the potential changes and the particle now occupies a metastable state relative to the new global minimum. As the motion is impeded by pinning barriers (blue), the particle creeps by thermal relaxation towards the new minimum. 
reaching the equilibrium state by warming, the creep motion of the vortex lattice reappears upon cooling. We capture this phenomenon within a model for creep in disordered systems.

The disappearance and reappearance of vortex creep in $2 \mathrm{H}-\mathrm{NbSe}_{2}$ is discussed and quantified in Sec. II. In Sec. III, we propose a generic model to describe the phenomenon of self-imposed creep. Section IV places in perspective the theoretical predictions with the experimental observations.

\section{VORTEX CREEP IN 2H-NbSe}

The layered compound $2 \mathrm{H}-\mathrm{NbSe}_{2}$ shows weak pinning and small creep rates [20,21]. Due to the material anisotropy, the properties of vortex creep depend on the field orientation relative to the crystallographic $c$ axis. To quantify this effect, we tilt the field angle (after cooling the system in a field $H=0.85 \mathrm{~T}$ ) by $\theta_{H}=70^{\circ}$ away from the $c$ axis.

$2 \mathrm{H}-\mathrm{NbSe}_{2}$ is an anisotropic superconductor, with the upper critical field varying as a function of the field direction and quantified by the anisotropy $\varepsilon \equiv H_{c 2, a b} / H_{c 2, c} \approx 1 / 3$. This leads to a distortion of the vortex lattice, discussed in detail in Refs. [22-29], which can be neglected for our purposes. A rotation of the magnetic field to the field angle $\theta_{H}$ induces creep, as the modification in the flux direction is counteracted by disorder present in the system. In isotropic systems, vortices would creep towards $\theta_{H}$; see Fig. 2. However, in anisotropic systems, the superconducting anisotropy modifies the angle for the equilibrium configuration of the vortex lattice to $\theta_{B}$. Minimization of the free energy yields [2,30-32]

$$
\sin \left(\theta_{B}-\theta_{H}\right)=\frac{H_{c 1}}{H} \frac{\left(1-\varepsilon^{2}\right) \sin \theta_{B} \cos \theta_{B}}{\left(\varepsilon^{2} \sin ^{2} \theta_{B}+\cos ^{2} \theta_{B}\right)^{1 / 2}},
$$

up to a logarithmic correction of the order of unity. In $2 \mathrm{H}-\mathrm{NbSe}_{2}$, using $H_{c 1}(T=0) \approx 200 \mathrm{G}$ for the lower critical field, we find $\theta_{B}-\theta_{H} \approx 0.8^{\circ}$, i.e., vortices are more inclined towards the $a b$ plane than the external field; see Fig. 2(a). In the regime where pinning is weak, i.e., where the Bean length [33] $\ell_{B} \approx c B / 4 \pi j_{c}$ is larger than the sample width $w$ and thickness $d \lesssim w$, vortices in the critical state [33,34] are straight and oriented along the angle $\theta_{B}^{c}=\theta_{B}-\left(w / 2 \ell_{B}\right) \sin \theta_{B}<$ $\theta_{B}$; see Fig. 2(a). With $j_{c} \sim 10^{3} \mathrm{~A} / \mathrm{cm}^{2}$, this critical angle deviates from the equilibrium angle $\theta_{B}$ as $\theta_{B}-\theta_{B}^{c} \approx 0.5^{\circ}$. We further notice that $\theta_{B}(T)$ dominantly depends on the current distribution via the penetration depth $\lambda(T)$ and is reflected in the above equation through $H_{c 1}(T)$. For small temperature changes $H_{c 1}(T)=H_{c 1}\left(T_{0}\right)+\delta H_{c 1}$, large fields $H \gg H_{c 1}$, and a tilt angle $\theta_{B}$ away from 0 or $\pi / 2$, the angle changes to $\theta_{B}(T)=\theta_{B}\left(T_{0}\right)-\delta \theta_{B}$, with

$$
\delta \theta_{B} \approx-\left[\theta_{B}\left(T_{0}\right)-\theta_{H}\right] \delta H_{c 1} / H_{c 1}\left(T_{0}\right) .
$$

The magnitude of this effect can be estimated using the Ginzburg-Landau scaling $H_{c 1}(T) \approx H_{c 1}(0)\left(1-T / T_{c}\right)$, which provides a relative change in $H_{c 1}$ between the experiment's low $\left(T_{0}=150 \mathrm{mK}\right)$ and high $(T=2 \mathrm{~K})$ temperatures of $\delta H_{c 1} / H_{c 1} \approx-0.29$, and hence $\delta \theta_{B} \approx 0.3^{\circ}$.

To investigate vortex creep in this system, we use a dilution-refrigerator scanning tunneling microscope (STM) in a three-axis vector magnet [35]. The cleaved $2 \mathrm{H}-\mathrm{NbSe}_{2}$ sample $\left(\sim 1 \times 1 \times 0.2 \mathrm{~mm}^{3}\right)$-grown with iodine vapor deposition—is studied with a gold tip, sharpened and cleaned (a)

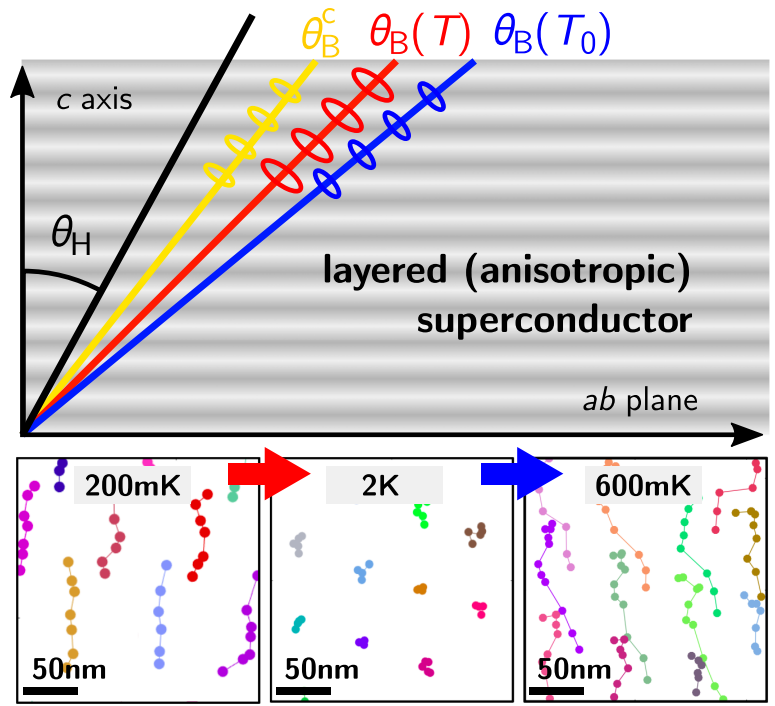

(c)
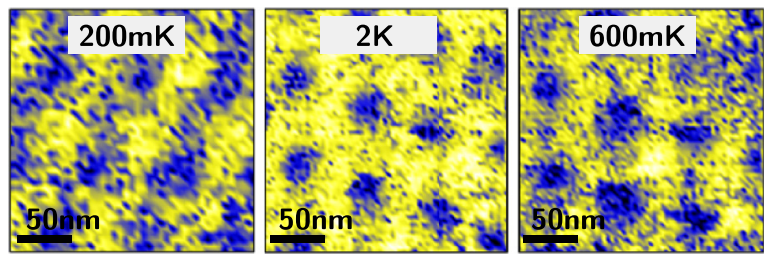

FIG. 2. (a) Schematic vortex alignment for an anisotropic superconductor in a tilted magnetic field $\left(\theta_{H}\right.$, black). The $T$-dependent current patterns-indicated as rings-define different equilibrium orientations $\theta_{B}$ at low $\left(T_{0}\right.$, blue) and higher $\left(T>T_{0}\right.$, red) temperature. When tilting the magnetic field to $\theta_{H}$, vortices creep towards the equilibrium angle $\theta_{B}^{c}<\theta_{B}\left(T_{0}\right)$ (yellow). (b) Vortex positions extracted from individual STM images (points) recorded at fixed temperature. Each vortex (core size $\xi \approx 7 \mathrm{~nm}$ ) is identified by a color and the lines join the position between two consecutive images. At low $T$, out-of-equilibrium vortices move between subsequent frames. The motion stops upon warming to $2 \mathrm{~K}$. At this temperature, vortices are fixed in a lattice. When cooling, motion reappears again and the average image shows that vortices do not stay at the same position in subsequent steps. (c) Time average of the series of STM images used in (b).

in situ [36]. At the field strength $H=0.85 \mathrm{~T}(H \| c)$, the magnetization is practically reversible so that no vortex motion is measured within days. Vortex motion only appears when rotating the field towards the layers (after field cooling to the base temperature $T_{0}=150 \mathrm{mK}$ with the magnetic field directed along the $c$ axis).

While specific vortex positions can be identified from an individual STM image (taking 23 minutes to record), consecutive images allow one to measure vortex motion over time. Figures 2(b) and 2(c) show the evolution of vortex positions at three fixed temperatures and the corresponding time-averaged STM image, respectively. It highlights the presence of vortex creep at low temperature, its disappearance upon warming, and its reappearance upon cooling. Note that vortices always move along the direction of the component of the magnetic field within the layers. In agreement with earlier reports [20,37-39], we observe a weak modulation of the vortex velocity when vortices travel by a distance of the order of the intervortex separation. The overall vortex displacement 


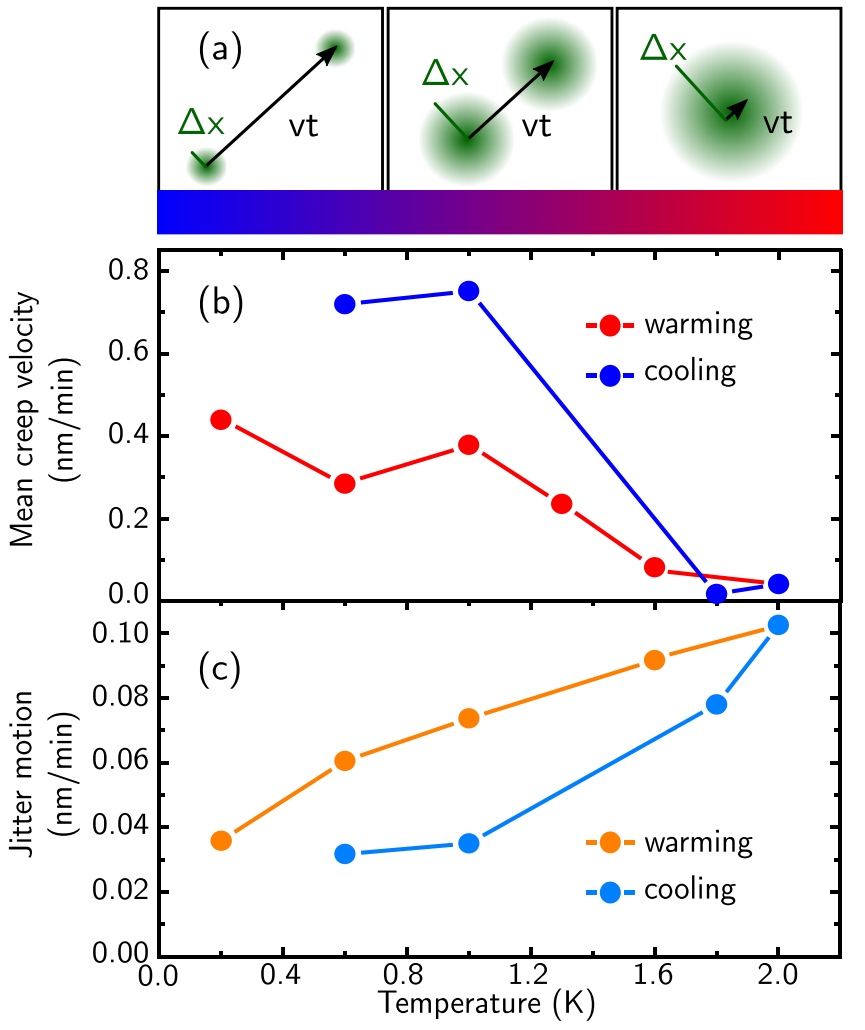

FIG. 3. (a) Schematic mean creep velocity (black arrow) and jitter motion (green cloud) for different temperatures. (b) Average creep velocity of vortices in $2 \mathrm{H}-\mathrm{NbSe}_{2}$ at tilted field and fixed temperature, recorded on a warming (red) and cooling (blue). (c) Standard deviation of the vortex jitter motion upon warming (orange) and cooling (light blue). The behavior agrees with the phenomenology of self-imposed creep.

translates to an average angular velocity $\dot{\theta}_{B} \approx 10^{-3} \mathrm{deg} / \mathrm{h}$ of vortices tilting to new equilibrium positions [see Fig. 2(a)] and the apparent absence of a measurable decay time within the observation window $(\sim 5 \mathrm{~h})$ is consistent with the lower bound $\left|\theta_{B}-\theta_{B}^{c}\right| / \dot{\theta}_{B} \gtrsim 100 \mathrm{~h}$ provided by the angular misalignment discussed above.

Without reinitializing the vortex state, imaging is repeated at different temperatures, while the system is kept at finite field strength and angle. Thus, temperature determines the preparation of the vortex state, as in Refs. [40-42]. Figure 3(a) schematically shows how the vortex displacement and jitter motion evolve upon changing temperature. In Figs. 3(b) and $3(\mathrm{c})$, the evolution of the average creep velocity $v(T)$ and jitter motion $\Delta x(T)$ is shown for a warming (red) and cooling (blue) cycle. Each data point is obtained from a series of STM frames. To find $v(T)$, we determine the position $\boldsymbol{r}_{j}^{i}$ of vortex $j$ in frame $i$ and evaluate the average displacement for each vortex per frame, given by $\delta r_{j}=\left|\boldsymbol{r}_{j}^{n_{j}}-\boldsymbol{r}_{j}^{1}\right| /\left(n_{j}-1\right)$, where $n_{j}$ denotes the number of frames where the $j$ th vortex appears. Averaging over all $N_{v}$ vortices for a given temperature, we arrive at the average creep velocity $v(T)=\left(t_{\mathrm{f}} N_{v}\right)^{-1} \sum_{j=1}^{N_{v}} \delta r_{j}$, with $t_{\mathrm{f}}$ the time for measuring one frame. To quantify the jitter motion, we evaluate the average jitter displacement $\delta s_{j}=$ $\left(\frac{1}{n_{j}-1} \sum_{i=2}^{n_{j}}\left|\delta \boldsymbol{r}_{j}^{i}\right|\right)-\delta r_{j}$, with $\left|\delta \boldsymbol{r}_{j}^{i}\right|$ the vortex displacement between two subsequent frames $i-1$ and $i$. The average over all frames at a fixed temperature now provides the jitter motion, $\Delta x(T)=N_{v}^{-1} \sum_{j=1}^{N_{v}} \delta s_{j}$.

The average creep velocity decreases upon warming and vanishes above $2 \mathrm{~K}$. Upon cooling, however, a finite velocity reappears. If the vortices were to reach a temperatureindependent minimum upon warming, the jitter motion would decrease upon cooling without a reappearance of creep motion. The reversible directed vortex motion upon thermal cycling is therefore a clear signature of self-imposed creep.

\section{MODEL FOR SELF-IMPOSED CREEP}

To capture the main observations, we discuss a simple, yet quite generic model for creep in disordered landscapes. First, let us note that motion is driven by thermal fluctuations, through an Arrhenius-type activation process across the pinning barriers. Then, the timescale $t \sim \tau \exp \left(U_{0} / k_{B} T\right)$ for thermal activation is determined by (i) the temperature $T$, (ii) the energy barrier $U_{0}$, and (iii) a microscopic timescale $\tau=\omega^{-1}$ ( $\omega$ is an attempt frequency to overcome the barrier). Creep is observed when the timescale of the experiment is such that $U_{0} \sim k_{B} T \ln (\omega t)$. To see the consequences of modifying the equilibrium, let us consider the problem of a particle confined in a one-dimensional parabolic trap $V_{0}(x)=k x^{2} / 2$ and subject to a driving force $V_{d}(x)=-F x$. To map the results of our simple model to a many-body system, we associate $x$ to a macroscopic observable, such as the position (or angle) of the vortex lattice for a given field direction. The force produces a drive towards the equilibrium position $\bar{x}=F / k$. In addition, we consider a disorder landscape $V_{p}(x)$ characterized by a typical depth $U_{0}$ and width $\xi$, with $k \xi^{2} / U_{0} \ll 1$; see Fig. 1 . We approximate the bare potential between neighboring minima $x_{ \pm}=x \pm \xi$ by $V_{p}(x+\delta) \approx\left[1-(\delta / \xi)^{2}\right] U_{0}$. The overall potential $V(x)=V_{0}+V_{d}+V_{p}$ features local minima in the range $\bar{x}-2 U_{0} / k \xi<x<\bar{x}+2 U_{0} / k \xi$, and the position-dependent activation barrier $U_{b}(x)$ to move from $x_{-}$to $x_{+}$(we assume $x<\bar{x}$ ) is

$$
U_{b}(x)=[(x-\bar{x}) k \xi]^{2} / 4 U_{0}+U_{0}+(x-\bar{x}) k \xi .
$$

The thermally activated motion in the opposite direction, i.e., from $x_{+}$to $x_{-}$, is penalized by an additional energy $-2(x-\bar{x}) k \xi>0$. A particle initially far from the minimum $\bar{x}$ will glide down the potential until reaching $\bar{x}-2 U_{0} / k \xi$ from where it will be thermally activated across ever-growing barriers. After a time $t$, the particle has reached a position $x_{T}$ satisfying Arrhenius' condition

$$
U_{b}\left(x_{T}\right)=k_{B} T \ln (\omega t),
$$

as smaller barriers have been overcome in exponentially shorter activation times. Inserting Eq. (3) into the condition (4), we obtain

$$
x_{T}=\bar{x}-\left(2 U_{0} / k \xi\right)\left[1-\sqrt{\left(k_{B} T / U_{0}\right) \ln (\omega t)}\right] .
$$

If $x_{T}$ is still far from $\bar{x}$ in the sense $\bar{x}-x_{T} \gg k_{B} T \ln (\omega t) / k \xi$ [translating to $T \ll T_{b} \equiv\left(U_{0} / k_{B}\right) / \ln (\omega t)$ ], the particle moves with an average velocity $v \approx 2 \xi / t$. If, however, the particle has relaxed in the vicinity of the global minimum, the thermal activation becomes almost equally probable in both directions. 


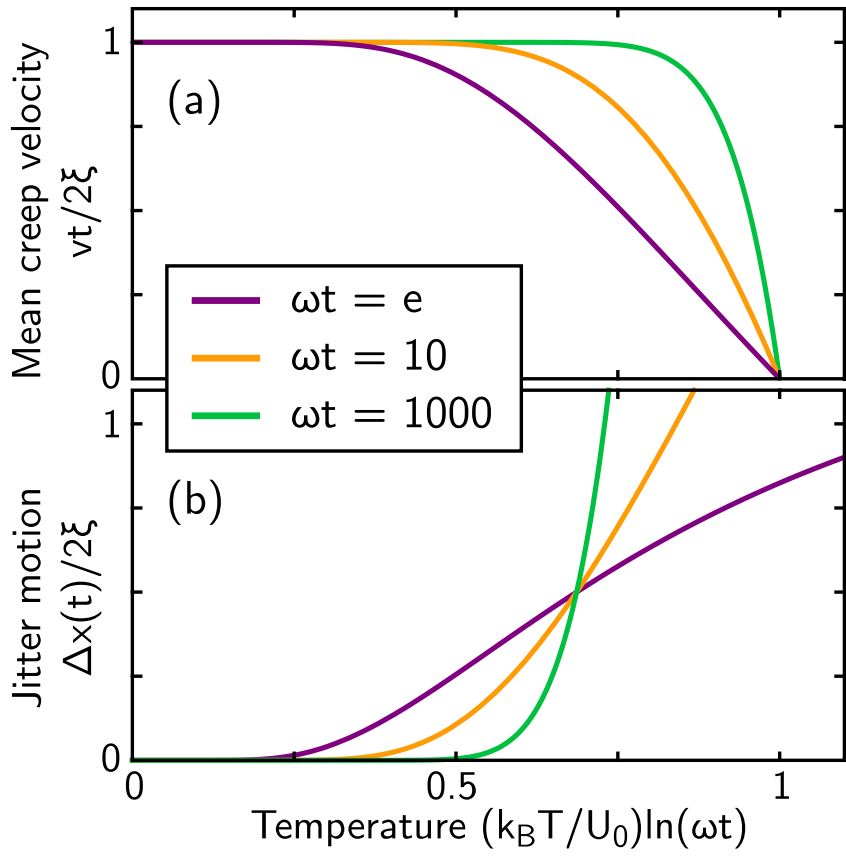

FIG. 4. (a) Average creep velocity $v$ (in units of $2 \xi / t$ ) as a function of temperature [see Eq. (6)] for different timescales $\omega t$. When temperature affects both the activation dynamics and the global potential minimum (see Fig. 1), the velocity profile is traced reversibly upon warming and cooling. (b) Standard deviation $\Delta x$ of the mean particle displacement, quantifying the isotropic thermal motion, or jitter; see Eq. (7).

Accounting for this bidirectional, yet asymmetric motion, we find a net average creep velocity

$$
v \approx \frac{2 \xi}{t}\left(1-\exp \left\{-\frac{4 U_{0}}{k_{B} T}\left[1-\sqrt{k_{B} T \ln (\omega t) / U_{0}}\right]\right\}\right) .
$$

The temperature dependence of $v$ is shown in Fig. 4(a) for different values $\omega t$. It is interesting to note that although the thermal activation energy increases upon warming, the creep velocity decreases. The validity of the above result is limited to temperatures $T<T_{b}$. For larger temperatures, the disorder landscape becomes irrelevant, as the particle relaxes to the global minimum within a time $\sim \omega^{-1}$.

The directed relaxation via creep [Eq. (6)] is blurred by an isotropic contribution, or jitter motion with zero mean and amplitude (standard deviation) given by

$$
\Delta x \sim \xi \sqrt{\omega t} \exp \left\{-\frac{2 U_{0}}{k_{B} T}\left[1-\sqrt{k_{B} T \ln (\omega t) / U_{0}}\right]\right\} .
$$

This result is obtained by describing the (forward-backward) activation across the barriers as a stochastic process (randomwalk motion), where the variance $(\Delta x)^{2}=\xi^{2} \omega_{\mathrm{rw}} t$ of the displacement grows linearly in time and is determined by the random-walk attempt frequency $\omega_{\text {rw }}=\omega \exp \left[2\left(x_{T}-\right.\right.$ $\left.\bar{x}) k \xi / k_{B} T\right]$. The exponent only depends on the difference between the barrier heights for forward and backward motion. This jitter motion persists beyond the disappearance of creep motion and reaches a similar magnitude when $k_{B} T \ln (\omega t) \sim$ $U_{0}$, as shown in Fig. 4(b).
When changing temperature, one has two distinguishable cases: one where the global minimum $\bar{x}$ is constant, and one where $\bar{x}(T)$ depends on $T$ through a temperature-dependent force $F(T)$. In the first case, the average creep velocity is given by the local disorder landscape seen by the particle and hence follows Eq. (6). For $T>T_{b}$, the system is fully relaxed and the particle reaches $\bar{x}$. Meanwhile, the magnitude of the jitter motion continuously increases; see Fig. 4(b). Subsequent cooling lowers the thermal energy and the particle's motion freezes in place at $\bar{x}$. This is the conventional response expected for creep. In the second case, when $\bar{x}$ depends on $T$ through a temperature-dependent force $F(T)$, the situation is drastically different. A temperature change $\Delta T$ imposes a shift $\bar{x}(T+\Delta T)-\bar{x}(T) \gg \xi$ of the global equilibrium position. Upon warming, the velocity and jitter motion are similar to the previous case. However, the values of $x(t)$ are different and, upon cooling, triggers particle motion tracing back (in magnitude) the warming curve, as schematically shown in Fig. 1. The appearance of creep upon cooling is caused by the two antagonistic roles of temperature-defining a $T$-dependent minimum and dictating the thermal relaxation towards it - and presents the most prominent manifestation of self-induced creep.

\section{DISCUSSION}

We compare our observations in $2 \mathrm{H}-\mathrm{NbSe}_{2}$ with the above model. The typical barrier to overcome during pinning by thermal fluctuations is given by Arrhenius' law $U_{0}=$ $k_{B} T \ln (\omega t)$. To observe both the equilibrium phase at high temperature and reentrant creep at low temperature, it is important that the temperature of the experiment is of the order of $\left(U_{0} / k_{B}\right) / \ln (\omega t)$. In contrast to the pinning energy of one defect site, here $U_{0}$ denotes the energy barrier for vortex creep $[43,44]$. Weak collective pinning theory $[1,2,45]$ provides the estimate $U_{0} \sim k_{B} T_{c}\left[\left(j_{c} / j_{\mathrm{dp}}\right)\left(B / H_{c 1}\right)^{3} / \mathrm{Gi}\right]^{1 / 2} v$, with the depairing current $j_{\mathrm{dp}}=c \Phi_{0} / 12 \sqrt{3} \pi^{2} \lambda^{2} \xi$, the GinzburgLevanyuk $[46,47]$ number $\mathrm{Gi} \sim\left[T_{c} / H_{c}(0)^{2} \xi^{3}\right]^{2}$, the condensation energy $H_{c}(0)^{2} \xi^{3}=\Phi_{0}^{2} \xi / 8 \pi^{2} \lambda^{2}$, and $v \sim 10^{-3}$ a small numerical number. From Refs. [48-53], we infer $j_{c} / j_{\mathrm{dp}} \sim$ $10^{-6}$ and $\mathrm{Gi} \sim 8 \times 10^{-7}$ and obtain $U_{0} \sim 5 k_{B} T_{c}$. This orderof-magnitude estimate is compatible with $U_{0} \lesssim k_{B} T_{c} \ln (\omega t)$, provided $\omega t \approx 10^{3}-10^{4}$, which is slightly larger than the values considered above (Fig. 4). Finally, the temperaturedependent current pattern in the layered superconductor naturally provides the necessary force $F(T)$ to induce creep upon cooling a tilted vortex lattice. Given the simplicity of the one-dimensional model, the agreement is remarkable. All important features predicted by the model - the disappearance and reappearance of the directed motion, together with the temperature evolution of the jitter motion [behaviors of $v(T)$ and $\Delta x(T)$, shown in Figs. 3(b) and 3(c) and in Figs. 4(a) and 4(b)] — are found in the experiment.

Given that the experimental timescale spans several minutes, our observation $\omega t \approx 10^{3}-10^{4}$ suggest a value for $\omega$ of the order of one $\mathrm{Hz}$. While a route for accurate determination of this attempt frequency is still lacking, the estimate $\omega=\alpha_{L} / \eta$ for a single vortex depends on the vortex viscosity $\eta$ and on the Labusch parameter [54] $\alpha_{L}$ (which in turn 
relates to the averaged potential curvature [55]). An analysis assuming vibrations with large $k$ vectors reports frequency values in the range $10^{6}-10^{10} \mathrm{~Hz}$ [56]. In our case however, vortices are not isolated, but rather interact nonlocally with many vortices $[2,3,57-59]$. Low $k$ vectors, or wavelengths comparable to the sample size, lead to highly dispersive elastic moduli which modify the attempt frequency by orders of magnitude [58-60]. Similar to our observation, previous measurements of slow vortex dynamics have reported [61,62] very low-frequency values for thermal motion and creep. Creep rates observed in layered cuprate superconductors involve extremely large timescales, indicating the relevance of collective creep $[2,3,58,60]$. Thus, even if the attempt rate of individual vortices is large, the dynamics as a lattice involves rates that are many orders of magnitude smaller. The temperature is far from melting, thus favoring collective rather than a single-vortex dynamics [63,64]. It is this near-equilibrium configuration with ultrasmall collective dynamics that allows for the observed cooling-imposed creep in our experiments.

The creep discussed here is very slow and the vortex velocity does not decay within our experimental time. However, the creep rate $S=d \ln (j) / d \ln (t)$ can assume a sizeable value compatible with the suggested lower bound [21] $S>$ $\left(T / T_{c}\right) \mathrm{Gi}^{1 / 2}$. Actually, $2 \mathrm{H}-\mathrm{NbSe}_{2}$ is among the materials with the lowest creep rates, close to $\mathrm{MgB}_{2}$ [21]. Creep between metastable vortex states that occur near the order-disorder transition of the vortex lattice in $2 \mathrm{H}-\mathrm{NbSe}_{2}$ or related to domain formation of lattices with different orientations in $\mathrm{MgB}_{2}$ has been reported [65-67]. Motion then appears when modifying the relative strength of competing interactions, and it might well occur that the equilibrium configuration at some particular locations is influenced by temperature. Collective motion is also found in the stochastic behavior of particle arrangements [11]. Depending on particle interactions, the dynamics transits from individual random motion to flocking. The timescale related to flocking motion shows a divergent behavior with increasing interaction. Other long-term dynamical behavior should appear in thermal effects and might lead to self-imposed creep whenever there are two or more parameters influencing the behavior of the system. Mixtures, such as alloys, concrete or rocks [68], liquids resolidifying under stress [12], steel under stress [13], colloidal stems, and magnetic domain walls or skyrmions [15] are cases of complex systems where self-imposed creep may be induced from temperature-dependent interactions.

The model of self-imposed creep explains the critical state dynamics in $2 \mathrm{H}-\mathrm{NbSe}_{2}$ at tilted magnetic fields; in particular, the commonly unexpected appearance of vortex motion when cooling. Likely, the balanced thermal activation dynamics and the temperature-dependent equilibrium could be matched in other complex systems.

\section{ACKNOWLEDGMENTS}

R.W. acknowledges the funding support from the Swiss National Science Foundation (SNSF) through the Early PostDoc.Mobility Fellowship. J.B.-L., E.H., I.G., and H.S. acknowledge support by the Spanish Research Agency (Grants No. FIS2017-84330-R, No. MDM-2014-0377 and No. RYC-2014-15093) and by the Comunidad de Madrid through program Nanomagcost-CM S2018/NMT-4321. I.G. acknowledges support by EU European Research Council PNICTEYES Grant Agreement No. 679080. J.A.G. and E.H. acknowledge support from the Departamento Administrativo de Ciencia, Tecnología e Innovación de Colombia, COLCIENCIAS (Programa de estancias postdoctorales convocatoria 784-2017 and Grant No. 120480863414) as well as from the Clúster de Investigación en Ciencias y Tecnologías Convergentes (NBIC) de la Universidad Central (Colombia). J.B.-L., J.A.G., E.H., I.G., and H.S. acknowledge SEGAINVEX-UAM. We wish to express a special thanks to Gianni Blatter and Vadim B. Geshkenbein for enlightening discussions and stimulating the interpretation of the experiments, and to Christian Spånslätt for carefully reading the manuscript.
[1] M. V. Feigel'man, V. B. Geshkenbein, A. I. Larkin, and V. M. Vinokur, Theory of Collective Flux Creep, Phys. Rev. Lett. 63, 2303 (1989).

[2] G. Blatter, M. V. Feigel'man, V. B. Geshkenbein, A. I. Larkin, and V. M. Vinokur, Vortices in high-temperature superconductors, Rev. Mod. Phys. 66, 1125 (1994).

[3] E. H. Brandt, The flux-line lattice in superconductors, Rep. Prog. Phys. 58, 1465 (1995).

[4] V. G. Kogan, M. Bullock, B. Harmon, P. Miranović, L. Dobrosavljević-Grujić, P. L. Gammel, and D. J. Bishop, Vortex lattice transitions in borocarbides, Phys. Rev. B 55, R8693 (1997).

[5] I. Guillamón, R. Córdoba, J. Sesé, J. M. De Teresa, M. R. Ibarra, S. Vieira, and H. Suderow, Enhancement of long-range correlations in a $2 \mathrm{~d}$ vortex lattice by an incommensurate $1 \mathrm{~d}$ disorder potential, Nat. Phys. 10, 851 (2014).

[6] J. Kierfeld, H. Nordborg, and V. M. Vinokur, Theory of Plastic Vortex Creep, Phys. Rev. Lett. 85, 4948 (2000).
[7] E. Dumont and A. C. Mota, Unconventional vortex dynamics in superconducting states with broken time-reversal symmetry, Phys. Rev. B 65, 144519 (2002).

[8] M. Konczykowski, C. J. van der Beek, M. A. Tanatar, H. Luo, Z. Wang, B. Shen, H. H. Wen, and R. Prozorov, Vortex creep and critical current densities in superconducting ( $\mathrm{Ba}, \mathrm{K}) \mathrm{Fe}_{2} \mathrm{As}_{2}$ single crystals, Phys. Rev. B 86, 024515 (2012).

[9] T. Klein, H. Grasland, H. Cercellier, P. Toulemonde, and C. Marcenat, Vortex creep down to $0.3 \mathrm{~K}$ in superconducting $\mathrm{Fe}(\mathrm{Te}, \mathrm{Se})$ single crystals, Phys. Rev. B 89, 014514 (2014).

[10] E. Herrera, J. Benito-Llorens, U. S. Kaluarachchi, S. L. Bud'ko, P. C. Canfield, I. Guillamón, and H. Suderow, Vortex creep at very low temperatures in single crystals of the extreme type-II superconductor $\mathrm{Rh}_{9} \mathrm{In}_{4} \mathrm{~S}_{4}$, Phys. Rev. B 95, 134505 (2017).

[11] N. W. Bode, D. W. Franks, and A. J. Wood, Making noise: Emergent stochasticity in collective motion, J. Theor. Biol. 267, 292 (2010). 
[12] B. J. Landrum, W. B. Russel, and R. N. Zia, Delayed yield in colloidal gels: Creep, flow, and re-entrant solid regimes, J. Rheol. 60, 783 (2016).

[13] D. E. Newland, On the time-dependent spin creep of a railway wheel, J. Mech. Eng. Sci. 24, 55 (1982).

[14] S. DuttaGupta, S. Fukami, C. Zhang, H. Sato, M. Yamanouchi, F. Matsukura, and H. Ohno, Adiabatic spin-transfer-torqueinduced domain wall creep in a magnetic metal, Nat. Phys. 12, 333 (2015).

[15] C. Zhou, C. Reichhardt, C. J. Olson Reichhardt, and I. J. Beyerlein, Dynamic phases, pinning, and pattern formation for driven dislocation assemblies, Sci. Rep. 5, 8000 (2015).

[16] R. Willa, A. E. Koshelev, I. A. Sadovskyy, and A. Glatz, Strongpinning regimes by spherical inclusions in anisotropic type-II superconductors, Supercond. Sci. Technol. 31, 014001 (2018).

[17] R. Willa, A. E. Koshelev, I. A. Sadovskyy, and A. Glatz, Peak effect due to competing vortex ground states in superconductors with large inclusions, Phys. Rev. B 98, 054517 (2018).

[18] I. A. Sadovskyy, A. E. Koshelev, W.-K. Kwok, U. Welp, and A. Glatz, Targeted evolution of pinning landscapes for large superconducting critical currents, Proc. Natl. Acad. Sci. USA 116, 10291 (2019).

[19] S. R. Foltyn, L. Civale, J. L. MacManus-Driscoll, Q. X. Jia, B. Maiorov, H. Wang, and M. Maley, Materials science challenges for high-temperature superconducting wire, Nat. Mater. 6, 631 (2007).

[20] A. M. Troyanovski, J. Aarts, and P. H. Kes, Collective and plastic vortex motion in superconductors at high flux densities, Nature (London) 399, 665 (1999).

[21] S. Eley, M. Miura, B. Maiorov, and L. Civale, Universal lower limit on vortex creep in superconductors, Nat. Mater. 16, 409 (2017).

[22] H. F. Hess, C. A. Murray, and J. V. Waszczak, ScanningTunneling-Microscopy Study of Distortion and Instability of Inclined Flux-Line-Lattice Structures in the Anisotropic Superconductor 2H-NbSe 2 , Phys. Rev. Lett. 69, 2138 (1992).

[23] H. F. Hess, C. A. Murray, and J. V. Waszczak, Flux lattice and vortex structure in $2 \mathrm{H}-\mathrm{NbSe}_{2}$ in inclined fields, Phys. Rev. B 50, 16528 (1994).

[24] P. L. Gammel, D. A. Huse, R. N. Kleiman, B. Batlogg, C. S. Oglesby, E. Bucher, D. J. Bishop, T. E. Mason, and K. Mortensen, Small Angle Neutron Scattering Study of the Magnetic Flux-Line Lattice in Single Crystal $2 \mathrm{H}-\mathrm{NbSe}_{2}$, Phys. Rev. Lett. 72, 278 (1994).

[25] L. J. Campbell, M. M. Doria, and V. G. Kogan, Vortex lattice structures in uniaxial superconductors, Phys. Rev. B 38, 2439 (1988).

[26] I. Fridman, C. Kloc, C. Petrovic, and J. Y. T. Wei, Lateral imaging of the superconducting vortex lattice using Dopplermodulated scanning tunneling microscopy, Appl. Phys. Lett. 99, 192505 (2011).

[27] I. Fridman, C. Kloc, C. Petrovic, and J. Y. T. Wei, Observation of an in-plane vortex lattice transition in the multiband superconductor $2 \mathrm{H}-\mathrm{NbSe}_{2}$ using scanning tunneling spectroscopy, arXiv:1303.3559.

[28] J. A. Galvis, E. Herrera, C. Berthod, S. Vieira, I. Guillamón, and $\mathrm{H}$. Suderow, Tilted vortex cores and superconducting gap anisotropy in $2 \mathrm{H}-\mathrm{NbSe}_{2}$, Commun. Phys. 1, 30 (2018).
[29] V. G. Kogan, L. N. Bulaevskii, P. Miranović, and L. Dobrosavljević-Grujić, Vortex-induced strain and flux lattices in anisotropic superconductors, Phys. Rev. B 51, 15344 (1995).

[30] A. V. Balatskii, L. I. Burlachkov, and L. P. Gor'kov, Magnetic properties of anisotropic type-II superconductors, Zh. Eksp. Teor. Fiz. 90, 1478 (1986) [Sov. Phys. JETP 63, 866 (1986)].

[31] V. G. Kogan, Uniaxial superconducting particle in intermediate magnetic fields, Phys. Rev. B 38, 7049 (1988).

[32] L. N. Bulaevskii, Anomalous behavior of torque at high angles in high-temperature superconductors, Phys. Rev. B 44, 910 (1991).

[33] C. P. Bean, Magnetization of Hard Superconductors, Phys. Rev. Lett. 8, 250 (1962).

[34] R. Willa, V. B. Geshkenbein, and G. Blatter, Campbell penetration in the critical state of type-II superconductors, Phys. Rev. B 92, 134501 (2015).

[35] J. A. Galvis, E. Herrera, I. Guillamón, J. Azpeitia, R. F. Luccas, C. Munuera, M. Cuenca, J. A. Higuera, N. Díaz, M. Pazos, M. García-Hernandez, A. Buendía, S. Vieira, and H. Suderow, Three axis vector magnet set-up for cryogenic scanning probe microscopy, Rev. Sci. Instrum. 86, 013706 (2015).

[36] J. G. Rodrigo, H. Suderow, S. Vieira, E. Bascones, and F. Guinea, Superconducting nanostructures fabricated with the scanning tunneling microscope, J. Phys.: Condens. Matter 16, R1151 (2004).

[37] A. M. Troyanovski, M. van Hecke, N. Saha, J. Aarts, and P. H. Kes, STM Imaging of Flux Line Arrangements in the Peak Effect Regime, Phys. Rev. Lett. 89, 147006 (2002).

[38] I. Guillamón, H. Suderow, S. Vieira, J. Sesé, R. Córdoba, J. M. De Teresa, and M. R. Ibarra, Direct Observation of Stress Accumulation and Relaxation in Small Bundles of Superconducting Vortices in Tungsten Thin Films, Phys. Rev. Lett. 106, 077001 (2011)

[39] J. Galvis, E. Herrera, I. Guillamón, S. Vieira, and H. Suderow, Vortex cores and vortex motion in superconductors with anisotropic Fermi surfaces, Physica C 533, 2 (2017).

[40] Y. Paltiel, E. Zeldov, Y. N. Myasoedov, H. Shtrikman, S. Bhattacharya, M. J. Higgins, Z. L. Xiao, E. Y. Andrei, P. L. Gammel, and D. J. Bishop, Dynamic instabilities and memory effects in vortex matter, Nature (London) 403, 398 (2000).

[41] R. Willa, V. B. Geshkenbein, R. Prozorov, and G. Blatter, Campbell Response in Type-II Superconductors Under Strong Pinning Conditions, Phys. Rev. Lett. 115, 207001 (2015).

[42] R. Willa, M. Marziali Bermúdez, and G. Pasquini, Thermal hysteresis of the Campbell response as a probe for bulk pinning landscape spectroscopy, Phys. Rev. B 98, 184520 (2018).

[43] M. Buchacek, R. Willa, V. B. Geshkenbein, and G. Blatter, Persistence of pinning and creep beyond critical drive within the strong pinning paradigm, Phys. Rev. B 98, 094510 (2018).

[44] M. Buchacek, R. Willa, V. B. Geshkenbein, and G. Blatter, Strong pinning theory of thermal vortex creep in type-II superconductors, Phys. Rev. B 100, 014501 (2019).

[45] A. I. Larkin and Y. N. Ovchinnikov, Pinning in type-II superconductors, J. Low Temp. Phys. 34, 409 (1979).

[46] A. P. Levanyuk, Contribution to the theory of light scattering near the second-order phase-transition points, Zh. Eksp. Teor Fiz. 36, 810 (1959) [Sov. Phys. JETP 36, 571 (1959)]. 
[47] V. L. Ginzburg, Some remarks on phase transitions of second kind and the microscopic theory of ferroelectric materials, Fiz. Tverd. Tela (Leningrad) 2, 2031 (1960) [Sov. Phys. Solid State 2, 1824 (1961)].

[48] S. S. Banerjee, N. G. Patil, S. Saha, S. Ramakrishnan, A. K. Grover, S. Bhattacharya, G. Ravikumar, P. K. Mishra, T. V. Chandrasekhar Rao, V. C. Sahni, M. J. Higgins, E. Yamamoto, Y. Haga, M. Hedo, Y. Inada, and Y. Onuki, Anomalous peak effect in $\mathrm{CeRu}_{2}$ and $2 \mathrm{H}-\mathrm{NbSe}_{2}$ : Fracturing of a flux line lattice, Phys. Rev. B 58, 995 (1998).

[49] M. Menghini, Y. Fasano, and F. de la Cruz, Critical current and topology of the supercooled vortex state in $\mathrm{NbSe}_{2}$, Phys. Rev. B 65, 064510 (2002).

[50] G. Pasquini, D. P. Daroca, C. Chiliotte, G. S. Lozano, and V. Bekeris, Ordered, Disordered, and Coexistent Stable Vortex Lattices in $\mathrm{NbSe}_{2}$ Single Crystals, Phys. Rev. Lett. 100, 247003 (2008).

[51] S. Mohan, J. Sinha, S. S. Banerjee, A. K. Sood, S. Ramakrishnan, and A. K. Grover, Large Low-Frequency Fluctuations in the Velocity of a Driven Vortex Lattice in a Single Crystal of $2 \mathrm{H}-\mathrm{NbSe}_{2}$ Superconductor, Phys. Rev. Lett. 103, 167001 (2009).

[52] A. Maldonado, S. Vieira, and H. Suderow, Supercurrent on a vortex core in $2 \mathrm{H}-\mathrm{NbSe}_{2}$ : Current-driven scanning tunneling spectroscopy measurements, Phys. Rev. B 88, 064518 (2013).

[53] S. Eley, K. Khilstrom, R. Fotovat, Z. L. Xiao, A. Chen, D. Chen, M. Leroux, U. Welp, W. K. Kwok, and L. Civale, Glassy dynamics in a heavy ion irradiated $\mathrm{NbSe}_{2}$ crystal, Sci. Rep. 8, 13162 (2018).

[54] R. Labusch, Calculation of the critical field gradient in type-II superconductors, Cryst. Lattice Defects 1, 1 (1969).

[55] R. Willa, V. B. Geshkenbein, and G. Blatter, Probing the pinning landscape in type-II superconductors via Campbell penetration depth, Phys. Rev. B 93, 064515 (2016).

[56] E. Brandt, Thermal fluctuation of the vortex positions in highTc superconductors, Physica C: Superconduct. Appl. 162-164, 1167 (1989).

[57] E. H. Brandt, Penetration of Magnetic ac Fields into Type-II Superconductors, Phys. Rev. Lett. 67, 2219 (1991).

[58] Y. Yeshurun, A. P. Malozemoff, and A. Shaulov, Magnetic relaxation in high-temperature superconductors, Rev. Mod. Phys. 68, 911 (1996).
[59] E. H. Brandt, Statics and dynamics of the vortex lattice in highTc superconductors, J. Low Temp. Phys. 139, 21 (2005).

[60] M. V. Feigel'man and V. M. Vinokur, Thermal fluctuations of vortex lines, pinning, and creep in high- $T_{c}$ superconductors, Phys. Rev. B 41, 8986 (1990).

[61] B. Raes, C. C. de Souza Silva, A. V. Silhanek, L. R. E. Cabral, V. V. Moshchalkov, and J. Van de Vondel, Closer look at the low-frequency dynamics of vortex matter using scanning susceptibility microscopy, Phys. Rev. B 90, 134508 (2014).

[62] C. C. de Souza Silva, B. Raes, J. Brisbois, L. R. E. Cabral, A. V. Silhanek, J. Van de Vondel, and V. V. Moshchalkov, Probing the low-frequency vortex dynamics in a nanostructured superconducting strip, Phys. Rev. B 94, 024516 (2016).

[63] O. M. Auslaender, L. Luan, E. W. J. Straver, J. E. Hoffman, N. C. Koshnick, E. Zeldov, D. A. Bonn, R. Liang, W. N. Hardy, and K. A. Moler, Mechanics of individual isolated vortices in a cuprate superconductor, Nat. Phys. 5, 35 (2009).

[64] L. Embon, Y. Anahory, A. Suhov, D. Halbertal, J. Cuppens, A. Yakovenko, A. Uri, Y. Myasoedov, M. L. Rappaport, M. E. Huber, A. Gurevich, and E. Zeldov, Probing dynamics and pinning of single vortices in superconductors at nanometer scales, Sci. Rep. 5, 7598 (2015).

[65] C. Rastovski, K. J. Schlesinger, W. J. Gannon, C. D. Dewhurst, L. DeBeer-Schmitt, N. D. Zhigadlo, J. Karpinski, and M. R. Eskildsen, Persistence of Metastable Vortex Lattice Domains in $\mathrm{MgB}_{2}$ in the Presence of Vortex Motion, Phys. Rev. Lett. 111, 107002 (2013).

[66] M. Marziali Bermúdez, M. R. Eskildsen, M. Bartkowiak, G. Nagy, V. Bekeris, and G. Pasquini, Dynamic Reorganization of Vortex Matter into Partially Disordered Lattices, Phys. Rev. Lett. 115, 067001 (2015).

[67] M. Marziali Bermúdez, E. R. Louden, M. R. Eskildsen, C. D. Dewhurst, V. Bekeris, and G. Pasquini, Metastability and hysteretic vortex pinning near the order-disorder transition in $\mathrm{NbSe}_{2}$ : Interplay between plastic and elastic energy barriers, Phys. Rev. B 95, 104505 (2017).

[68] Y. Zhang, D. C. Richardson, O. S. Barnouin, C. Maurel, P. Michel, S. R. Schwartz, R.-L. Ballouz, L. A. Benner, S. P. Naidu, and J. Li, Creep stability of the proposed AIDA mission target 65803 Didymos: I. Discrete cohesionless granular physics model, Icarus 294, 98 (2017). 